\title{
Weaning from mechanical ventilatory support in the 21st century: Procrustes' bed, rocket science, or art?
}

\author{
Dietrich Henzler, MD, PhD · Richard Hall, MD
}

Published online: 30 May 2009

(c) Canadian Anesthesiologists' Society 2009

Mechanical ventilatory support of respiratory system dysfunction has been a fundamental part of the provision of care to the critically ill patient for over five decades. Indeed, the development of the specialty of critical care medicine has occurred in parallel with the development of modes of mechanical ventilation and our understanding of its attendant (patho)physiology. Nevertheless, while we have learned a great deal about the use of mechanical ventilation, both for elective purposes in the operating room and for therapeutic purposes in the intensive care unit (ICU), the process of liberating a patient from mechanical ventilation ("weaning") remains less developed than initiating ventilatory support. We agree with Milic Emili, ${ }^{1}$ who wrote the Art of Weaning (deciding how and when a patient should begin the weaning process), that its success remains dependent on the experience, skills, and intuition of the treating physician.

In the absence of data to the contrary, (un)informed and/ or popular opinion is often a major determinant of practice in this area. For example, in 1975, Petty vigorously argued against the use of newly developed modes of assisted ventilation for weaning, such as intermittent mandatory ventilation (IMV). ${ }^{2}$ Instead, he favoured continuous mandatory ventilation, notably by "adding mechanical deadspace or increasing $\mathrm{F}_{\mathrm{I}} \mathrm{CO}_{2}[\ldots]$ to maintain $\mathrm{CO}_{2}$ homeostasis". The patient should not "be unduly burdened,

D. Henzler, MD, PhD $\cdot$ R. Hall, MD

Division of Critical Care Medicine, Dalhousie University and the Capital District Health Authority, Halifax, NS, Canada

R. Hall, MD ( $ه)$

Department of Anesthesia, Queen Elizabeth II Health

Sciences Centre, 1796 Summer Street, Room 5452 HI,

Halifax, NS, Canada

e-mail: rihall@dal.ca nor should the medical team rely on a ventilator set at the progressively lower rate to forestall disaster if the patient is not ready". Discontinuation from ventilation should therefore "require no special circuitry or ventilator modification, but does require considerable judgment on the part of the respiratory care team." Despite this opinion, by the late 1980s, as data about efficacy (reduced duration of mechanical ventilation and complication rates (e.g., barotrauma)) became available and with refinements in ventilator technology, IMV became defined as the nonpareil weaning technique. ${ }^{3}$

Almost 20 years later, Cohen noted that a team approach, with weaning teams rounding formally three times per week (!) for a total of 20-40 min, marked a significant improvement in patient weaning. ${ }^{4} \mathrm{He}$ concluded that "planning and organization on the process of weaning" had an impact on success. However, Cohen's paper was published before much was known about how the practice of weaning varied amongst critical care physicians in general. By this time, landmark studies by Marini had unveiled the limitations of IMV in unloading the patient's work of breathing. ${ }^{5}$

With further refinements in ventilator technology, the ability to provide pressure support came into vogue as a modality for mechanical ventilation and weaning. Because it was generally perceived as providing a more physiological mode of ventilation and permitted patients to be awake during mechanical ventilation, pressure support ventilation became the new preferred weaning method in many centres (including our own).

In a comprehensive review, Tobin ${ }^{6}$ summarized and classified the evolution of weaning techniques. He reasoned that weaning success predictors, techniques, and pathophysiologic understanding each showed a biphasic progress, with cross-fertilization and serendipity as important factors 
as science for their development. As these factors are hard to predict and even harder to control, a multitude of opinions about the best weaning methods have persisted and continue to be argued by their proponents. ${ }^{6}$

In this issue of the Journal, Burns et al. ${ }^{7}$ present the results from a national weaning survey amongst ICU physicians and respiratory therapists, in an effort to elucidate the murkiness around current weaning practice. Using rigorous methodology previously developed by the investigators, Canadian decision-makers in the weaning process were surveyed. As a result, Burns et al. concluded that weaning practice has changed over the years, but is relatively uniform in Canada.

This study raises three main questions: Has practice changed and is there a uniform practice? Are the results specific to Canada? Could there be a difference between perception and practice?

The answer to the first question is a qualified yes. In the study by Burns et al., ${ }^{7}$ use of pressure support as the preferred weaning method was reported in $>80 \%$ of respondents. An important component of variability in practice is the division of responsibility for weaning decision-making and success between caregivers. For example, physicians might decide differently from respiratory therapists about when and how a patient should be weaned from a ventilator; specialist intensivists might decide differently from in-training physicians; and anesthetists might decide differently from internists or surgeons. Unfortunately, this study is underpowered to answer these questions and no direct comparisons are available; however, everyone has experienced the influence that differences in specialty education and experience have on daily practice. It appears, in this regard, that we are relatively uniform in our weaning decision-making processes.

In the study by Burns et al., ${ }^{7}$ the physician groups surveyed were ICU medical directors. While there is no reason to believe a deliberate bias in their answers, it cannot be ignored that this is a selected group of respondents providing their personal viewpoints, which may or may not reflect the group practice.

Interventions proven effective in improving the weaning process were surveyed. The good news is that $>95 \%$ of respondents assess the readiness to wean from mechanical ventilation by conducting a spontaneous breathing trial (SBT) at least once daily. The bad news is that less than half of the units have a standardized written weaning guide. The most commonly employed method to facilitate weaning was pressure support (PS). However, PS delivers fixed support, independent from the patient's needs. It either overcompensates (does not promote weaning) or undercompensates (does not efficiently unload the patient's work of breathing), suggesting there remains significant room for improving the weaning process. Many attempts have been made to design closed-loop systems adapting to the patient's actual needs, such as proportional assist ventilation, adaptive support ventilation, or automatic tube compensation. Although these additions are available in commercial ventilators, their use is apparently grossly underutilized.

This leads directly to the second question: Is the weaning practice described in Canada similar to other populations? As Burns et al. ${ }^{7}$ acknowledge, the answer is almost certainly no. Other modes of partial ventilatory support might be employed, and different caregivers might be responsible for initiating and conducting the weaning process. For example, in Europe ${ }^{8}$ and worldwide, ${ }^{9}$ biphasic positive airway pressure is frequently used for weaning, but it is used rarely in this Canadian study. Furthermore, the authors found that, while the method for SBT preferred in Canada is a low level of pressure support, a recent observation revealed that $71 \%$ of respondents from 349 ICUs in 23 countries worldwide identified T-piece as the method of choice. $^{10}$

Where do these differences originate? Clearly, in this age of information technology, everybody has access to the same research results. Burns et al. make no attempt to explain this phenomenon, although the answer may lie with the clinicians involved in the decision-making during weaning. The system of dividing the work between physician and respiratory therapist is more or less unique to North America. Do physicians wean differently? Some results of the study might imply this (refer to the discussion on volume vs pressure modes), although no specific analysis could be completed in this regard. In fact, at least in our units, respiratory therapists spend more time at the bedside and, consequently, are more likely to be leading decisions about how to wean, while physicians are more likely to be leading decisions about when to wean and extubate the trachea.

Regarding the third question, due to inherent limitations of survey research, a conclusion cannot be reached regarding actual and perceived practice. The survey's merit lies in allowing us to appreciate the perceived practice in a described setting (which, unfortunately, may include responses based on what clinicians believe they ought to do rather than what they actually do). However, the German SepNet, a collaboration of clinicians dedicated to sepsis research, has demonstrated the inherent limitations in these observations. ${ }^{11}$ After surveying the practice, they conducted clinical audits and scrutinized the data. The results were impressive. For example, although $80 \%$ of respondents answered that they administered low-tidal volumes according to the Surviving Sepsis Campaign guidelines; in fact, more than $80 \%$ of patients received $>8 \mathrm{~mL} \cdot \mathrm{kg}^{-1}$ of tidal volume. 
When questioned about a specific weaning decision for a patient in ICU, often the selection was based in part on "a gut feeling" rather than based on evidence. This uncertainty is reflected in the reported outcomes of this survey. Although it may be comforting to know our colleagues struggle with the same problems, the issue regarding the correct weaning technique and its success still hovers between rocket science and magic.

More often than not, the answer is somewhere in the middle. On the one hand, the improved understanding of the physiology of weaning has clearly led us away from trying to adjust the patient to the ventilator. Doing so seems analogous to "Procrustes bed." 12 According to Greek mythology, Procrustes ("The Stretcher") was an antic villain who tortured travellers falling victim to him by adjusting them to fit the length of his bed; he either cut off overlapping limbs or stretched them to size.

On the other hand, there remains a large element of art applied to weaning methods. The broad-spectrum use of various methods suggests that successful weaning has become more of an applied science rather than a top-secret procedure only available to a select few.

Since this is a survey, nothing can be revealed about the actual success of current weaning practices. Following the theorem of Crick's Gossip test and Watson's Boredom principle, ${ }^{13}$ surveying falls into the mundane field of trendy science. However, there is an opportunity to extend these observations into paradigm-shifting revolutionary science, ${ }^{14}$ if further research reveals the superior weaning technique given the technology available today. The authors have assumed this responsibility, and we look forward to reading the results of their efforts in future publications.

\section{Le sevrage de la ventilation mécanique au $21^{\text {ème }}$ siècle: lit de Procruste, science exacte, ou art ?}

Depuis plus d'un demi-siècle, la ventilation mécanique comme traitement de support pour un dysfonctionnement de l'appareil respiratoire fait partie intégrante de la prestation de soins aux patients gravement malades. En fait, l'évolution de la spécialité de la médecine des soins intensifs s'est faite en parallèle au développement de divers modes de ventilation mécanique et de notre compréhension de la physio(patho)logie afférente. Toutefois, bien que nous ayons beaucoup appris au sujet de l'utilisation de la ventilation mécanique, qu'il s'agisse d'un contexte non urgent en salle d'opération ou pour des raisons thérapeutiques dans l'unité des soins intensifs (USI), le processus nécessaire pour libérer un patient de la ventilation mécanique (le « sevrage ») demeure un aspect moins développé que celui d'installer la ventilation mécanique. Nous sommes d'accord avec Milic Emili, ${ }^{1}$ auteur de « The Art of Weaning » (l'Art du sevrage) - ouvrage qui traite de la prise de décision du moment et de la manière dont un patient devrait commencer à être sevré ; selon Milic Emili, le succès du sevrage repose sur l'expérience, la compétence et l'intuition du médecin traitant.

En l'absence de données contraires, l'opinion (mal) informée et/ou populaire joue souvent un rôle prépondérant dans la pratique dans ce domaine. Par exemple, en 1975, Petty s'est résolument prononcé contre l'utilisation de modes de ventilation assistée nouvellement mis au point pour le sevrage, comme par exemple la ventilation obligatoire intermittente (VOI). ${ }^{2}$ Par contre, il a privilégié la ventilation obligatoire continue, notamment en « ajoutant un volume perdu mécanique ou en augmentant la $\mathrm{F}_{\mathrm{I}} \mathrm{CO}_{2}$ [...] afin de maintenir l'homéostasie du $\mathrm{CO}_{2}$ ». Le patient ne devrait pas «être excessivement accablé, et l'équipe médicale ne devrait pas compter sur un respirateur réglé à un taux de débit progressivement plus bas pour prévenir une catastrophe, si le patient n'est pas prêt ». Ainsi, l'interruption de la ventilation devrait « ne nécessiter aucun circuit spécial ou modification du respirateur, mais nécessite beaucoup de bon sens de la part de l'équipe de soins respiratoires ». Malgré cette opinion, à la fin des années 80, des données sont apparues concernant l'efficacité (durée réduite de la ventilation mécanique et taux de complications (par ex., barotraumatisme)) et la technologie des respirateurs a évolué : la VOI est devenue la technique incontournable du sevrage. ${ }^{3}$

Près de vingt ans plus tard, Cohen a remarqué qu'une approche en équipe, avec des équipes de sevrage faisant des tournées formelles trois fois par semaine (!) pour un total de 20 à $40 \mathrm{~min}$, améliorait significativement le sevrage des patients. ${ }^{4}$ Il conclut que « la planification et l'organisation du processus de sevrage »avaient des répercussions sur la réussite du processus. L'article de Cohen a cependant été publié avant que nous ne réalisions combien les pratiques de sevrage variaient entre les médecins des soins intensifs en général. À ce moment-là, les études jalon de Marini avaient révélé les limites de la VOI quant au soutien fourni par cette technique pour diminuer la travail respiratoire du patient pour respirer. ${ }^{5}$

Grâce aux avancées dans la technologie des ventilateurs, la capacité de procurer une aide inspiratoire est devenue à la mode en tant que modalité fournissant ventilation mécanique et sevrage. Cette méthode a été généralement perçue comme procurant un mode de ventilation plus physiologique et permet aux patients d'être éveillés pendant la ventilation mécanique ; par conséquent, l'aide inspiratoire est devenue la nouvelle méthode de sevrage préférée dans de nombreux centres (y compris le nôtre). 
Dans une revue documentaire étendue, Tobin ${ }^{6}$ a résumé et catégorisé l'évolution des techniques de sevrage. Selon son raisonnement, les indices de prédiction, les techniques et la compréhension physiopathologique d'un sevrage réussi montraient tous une évolution biphasique où l'enrichissement mutuel et le hasard jouaient un rôle aussi important que la science. Ces facteurs étant difficiles à prédire et encore plus difficiles à contrôler, les opinions concernant les meilleures méthodes de sevrage demeurent foison et continuent d'être discutées par leurs défenseurs. ${ }^{6}$

Dans ce numéro du Journal, Burns et coll. ${ }^{7}$ présentent les résultats d'un sondage national portant sur le sevrage, mené auprès des médecins des soins intensifs et des inhalothérapeutes, dont l'objectif était de faire la lumière sur l'obscurité entourant les pratiques de sevrage actuelles. À l'aide d'une méthodologie rigoureuse précédemment élaborée par les chercheurs, les auteurs ont interrogé les principaux acteurs du processus de sevrage au Canada. Burns et coll. concluent que la pratique de sevrage a évolué au fil des ans, mais qu'elle est relativement uniforme au Canada.

Cette étude soulève trois questions principales : La pratique a-t-elle changé et existe-t-il une pratique uniforme ? Les résultats sont-ils spécifiques au Canada ? Pourrait-il y avoir une différence entre la perception et la pratique ?

La réponse à la première de cette question est un oui prudent. Dans l'étude de Burns et coll., ${ }^{7}$ l'utilisation de l'aide inspiratoire comme méthode de sevrage préférée a été rapportée par $>80 \%$ des répondants. Un facteur important dans la variabilité des pratiques réside dans la responsabilité de décision et de succès de sevrage entre les différents prestataires de soins. Par exemple, les médecins pourraient prendre une décision différente de celle des inhalothérapeutes concernant le moment et la manière de sevrer un patient du respirateur ; les intensivistes spécialisés pourraient prendre une décision différente des médecins en formation ; et les anesthésistes pourraient prendre une décision différente de celle des internistes et des chirurgiens. Malheureusement, l'étude n'a pas la puissance statistique pour répondre à ces questions, et aucune comparaison directe n'est disponible ; toutefois, nous sommes tous conscients de l'influence d'une formation spécialisée et de l'expérience sur la pratique quotidienne. Par conséquent, il semble que nos processus de prise de décision de sevrage soient relativement uniformes.

Dans l'étude de Burns et coll., ${ }^{7}$ les groupes de médecins interrogés étaient des directeurs médicaux d'USI. Bien qu'il n'y ait aucune raison de remettre en question l'objectivité de leurs réponses, il faut cependant garder à l'esprit qu'il s'agit là d'un groupe choisi de répondants offrant leurs points de vue personnels, lesquels peuvent ou non être le reflet de la pratique de groupe.
Les interventions reconnues comme efficaces et améliorant le processus de sevrage ont été examinées. La bonne nouvelle, c'est que $>95 \%$ des répondants déterminent le bon moment pour procéder au sevrage de la ventilation en réalisant un test de ventilation spontanée au moins une fois par jour. La mauvaise nouvelle, c'est que moins de la moitié des unités disposaient de directives écrites standardisées de sevrage. L'aide inspiratoire était la méthode la plus couramment utilisée pour faciliter le sevrage. Toutefois, l'aide inspiratoire fournit un soutien fixe, indépendamment des besoins du patient. Cette méthode soit sur-compense (ne favorise pas le sevrage) ou souscompense (ne fournit pas un soutien efficace à la respiration du patient), ce qui suggère qu'il y a de la place pour une amélioration dans le processus de sevrage. Nombre de tentatives ont été faites pour concevoir des systèmes en circuit fermé s'adaptant aux besoins réels du patient, notamment la ventilation assistée proportionnelle, la ventilation assistée contrôlée, ou la compensation de sonde trachéale. Bien que ces fonctions supplémentaires soient disponibles sur les respirateurs commercialisés, il semble qu'elles soient largement sous-utilisées.

Ceci nous mène directement à la deuxième question : La pratique de sevrage décrite au Canada est-elle semblable aux autres populations? Tout comme Burns et coll. ${ }^{7}$ le reconnaissent, la réponse est très certainement non. D'autres modes d'aide inspiratoire partielle peuvent être utilisés, et d'autres prestataires de soins peuvent être en charge du début et de la réalisation du processus de sevrage. Par exemple, en Europe ${ }^{8}$ et dans le monde, ${ }^{9}$ la pression positive biphasique est souvent utilisée dans le cadre du sevrage, mais cette méthode est rarement utilisée dans cette étude canadienne. En outre, les auteurs ont découvert que, bien que la méthode préférée de test de ventilation spontanée au Canada consiste en un niveau bas d'aide inspiratoire, une observation récente a révélé que 71 $\%$ des répondants issus de 349 USI dans 23 pays dans le monde préféraient la méthode du tube en $\mathrm{T}^{10}$

D'où viennent ces différences ? Il est clair que dans notre ère des technologies de l'information, tout le monde a accès aux mêmes résultats de recherche. Burns et coll. ne tentent pas d'expliquer ce phénomène, bien que la réponse réside probablement chez les cliniciens qui prennent la décision de sevrer. Le système qui consiste à répartir le travail entre le médecin et l'inhalothérapeute est plus ou moins unique à l'Amérique du Nord. Les médecins utilisent-ils différentes méthodes pour procéder au sevrage ? Certains résultats de cette étude pourraient le suggérer (voir la discussion sur les modes en volume ou en pression), bien qu'aucune analyse spécifique n'ait pu être conclue à cet égard. En fait, du moins dans nos unités, les inhalothérapeutes passent plus de temps au chevet du patient; par conséquent, il est plus probable qu'ils mènent la discussion 
quant à savoir comment sevrer, alors que les médecins prendront plus facilement la décision de savoir quand sevrer et procéder à l'extubation trachéale.

Concernant la troisième question, en raison des limites inhérentes à la recherche par questionnaire, nous ne pouvons établir de conclusion quant à la pratique actuelle et perçue. Le mérite de ce sondage réside dans le fait qu'il nous donne un aperçu de la pratique perçue dans un contexte décrit (lequel sondage, malheureusement, pourrait comprendre des réponses fondées sur ce que les cliniciens croient qu'ils devraient faire plutôt que ce qu'ils font réellement). Néanmoins le réseau allemand SepNet, une collaboration de cliniciens qui se consacre à la recherche en sepsis, a démontré les limites inhérentes à ces observations. ${ }^{11}$ Après avoir évalué la pratique, ces chercheurs ont mené des contrôles cliniques et analysé les données ainsi obtenues. Les résultats étaient saisissants. Par exemple, bien que $80 \%$ des personnes interrogées aient répondu qu'elles administraient des volumes courants bas conformément aux directives du Surveying Sepsis Campaign, en fait, plus de $80 \%$ des patients ont reçu un volume courant de $>8 \mathrm{~mL} \cdot \mathrm{kg}^{-1}$.

Lorsque la question portait sur la décision spécifique de sevrer un patient de l'USI, la sélection se fondait souvent en partie sur «l'intuition » plutôt que sur des données probantes. Cette incertitude se reflète dans les devenirs rapportés de cette étude. Bien qu'il soit réconfortant de savoir que nos collègues sont aux prises avec le même type de problèmes que nous, la question de la méthode de sevrage correcte et du succès du sevrage oscille toujours entre science exacte et magie.

La plupart du temps, la réponse se trouve quelque part entre ces deux extrêmes. D'une part, l'amélioration de notre compréhension des mécanismes physiologiques impliqués dans le sevrage nous a éloigné de nos tentatives d'ajustement du patient au respirateur. Une telle démarche semble analogue au «lit de Procruste ». ${ }^{12}$ Dans la mythologie grecque, Procruste (« celui qui martèle pour allonger ») est un brigand qui torture les voyageurs qui tombent sous ses mains en les 'ajustant' afin qu'ils tiennent exactement dans le lit : soit il coupe les membres qui dépassent, soit il les étire jusqu'à la taille requise.

D'autre part, une composante artistique joue encore un rôle important dans les méthodes de sevrage. La diversité de méthodes suggère qu'un sevrage réussi est devenu davantage une science appliquée qu'une procédure top secrète connue uniquement de quelques heureux élus.

Étant donné qu'il s'agit ici d'un sondage, nous ne pouvons pas tirer de conclusions concernant le succès réel des pratiques actuelles de sevrage. Si l'on se fonde sur le principe du test de 'bavardage' de Crick et 'd'ennui' de Watson (c'est-à-dire, faire des recherches dans les domaines qu'on aime - dont on bavarde - et éviter les domaines qui nous ennuient), ${ }^{13}$ les sondages tombent dans le domaine banal de la science à la mode. Toutefois, ces observations pourraient être étendues pour être comprises dans le domaine de la science révolutionnaire qui modifie les paradigmes ${ }^{14}$ - si des recherches supplémentaires devaient révéler une technique de sevrage comme étant supérieure parmi les technologies disponibles actuellement. Les auteurs ont endossé cette responsabilité, et nous avons hâte de lire les résultats de ces efforts dans des publications futures.

Conflicts of interest Both Drs. Henzler and Hall are active members of the Canadian Critical Care Trials Group and participate in scientific investigations, including those conducted by or involving the study authors.

\section{References}

1. Milic-Emili J. Is weaning an art or a science? Am Rev Respir Dis 1986; 134: 1107-8.

2. Petty TL. Editorial: IMV vs IMC. Chest 1975; 67: 630-1.

3. Venus B, Smith RA, Mathru M. National survey of methods and criteria used for weaning from mechanical ventilation. Crit Care Med 1987; 15: 530-3.

4. Cohen IL. Weaning from mechanical ventilation-the team approach and beyond. Intensive Care Med 1994; 20: 317-8.

5. Marini JJ, Capps JS, Culver BH. The inspiratory work of breathing during assisted mechanical ventilation. Chest 1985; 87: 612-8.

6. Tobin MJ. Remembrance of weaning past: the seminal papers. Intensive Care Med 2006; 32: 1485-93.

7. Burns KE, Lellouche F, Loisel F, et al. Weaning critically ill adults from invasive mechanical ventilation: a national survey. Can J Anesth 2009; 56: 8. doi:10.1007/s12630-009-9124-8.

8. Staudinger T, Kordova H, Roggla M, et al. Comparison of oxygen cost of breathing with pressure-support ventilation and biphasic intermittent positive airway pressure ventilation. Crit Care Med 1998; 26: 1518-22.

9. Seymour CW, Frazer M, Reilly PM, Fuchs BD. Airway pressure release and biphasic intermittent positive airway pressure ventilation: are they ready for prime time? J Trauma 2007; 62: 1298308.

10. Esteban A, Ferguson ND, Meade MO, et al. Evolution of mechanical ventilation in response to clinical research. Am J Respir Crit Care Med 2008; 177: 170-7.

11. Brunkhorst FM, Engel C, Ragaller $M$, et al. Practice and perception-a nationwide survey of therapy habits in sepsis. Crit Care Med 2008; 36: 2719-25.

12. Dreyfuss D, Saumon G. Pressure-volume curves: searching for the grail or laying patients with adult respiratory distress syndrome on procrustes' bed? Am J Respir Crit Care Med 2001; 163: $2-3$.

13. Charlton BG. Crick's gossip test and Watson's boredom principle: a pseudo-mathematical analysis of effort in scientific research. Med Hypotheses 2008; 70: 1-3.

14. Kuhn TS. The Structure of Scientific Revolutions. 1st ed. Chicago: University of Chicago Press; 1962. 